\title{
Analysis of Uniform and Tapered CPW-fed Zigzag Monopoles Used as Feed Structures for Wide-Slot Radiators
}

\author{
Amartya BANERJEE, Sayan CHATTERJEE, Bhaskar GUPTA, Anup Kumar BANDYOPADHYAY \\ Dept. of ETCE, Jadavpur University, 188 Raja S. C. Mallick Road, Kolkata - 700032, India
}

amartyapotter@gmail.com, sayan1234@gmail.com,gupta_bh@yahoo.com, akbandyopadhyay@gmail.com

Submitted January 4, 2019 / Accepted June 5, 2019

\begin{abstract}
C P W$-fed zigzag monopole radiators can be used to excite wide slots etched on the CPW-grounds for gain enhancement purposes, without significantly altering its resonance. The circuit analysis for $C P W$-fed zigzag monopole acting as a feed to a wide square slot is presented. Later, the uniform zigzag feed is tapered to support a gradual propagation constant modification. In both cases, the zigzag feed is conceived as an array of two constituent monopoles fed from the same source with modified propagation constants. Simulations along with the measured results are provided to validate the theoretical work which showed less than 2\% error achieved in the predictions. Measured gains of the radiators are reported at their resonance frequencies, $2.93 \mathrm{~dB}$ and $3.19 \mathrm{~dB}$ respectively for the uniform and tapered zigzag feeds, which validates the observation of gain enhancement in this article.
\end{abstract}

\section{Keywords}

Circuit model analysis, closed-form expressions, uniform and tapered zigzag monopole, wide-slot radiator, gain enhancement

\section{Introduction}

Recently some theoretical investigations on CPW-fed monopole antennas are reported in literature [1-4], one of which is by the present authors that aims to model the monopoles into equivalent structures with propagation constant modifications [4]. Such monopole-like radiators possess near omni-directional radiation properties and are suitable for low gain antenna applications [4]. To enhance the gain-performances, it may be suggested to use the CPW-fed strip monopoles as excitations to wide regular slots etched on the CPW-grounds [5], [6]. With suitably chosen dimensions, such a technique will increase the effective aperture area of the concerned radiator, without significantly altering the resonant feed characteristics. This extension will lead to a better radiating performance and will improve the concerned structures for further practical operations. However, the theoretical analysis presented in the works [2-4] needs to be validated for such alterations in the structural aspects of the concerned shapes. This work aims to analyze CPW-fed strip monopole antennas used as excitations to wide slots etched out on the ground for gain enhancement purposes. In this context, the authors are influenced by the zigzag structure reported in [4] to be used and analyzed as an excitation to wide slot radiators. This analysis represents a zigzag structure by a suitable slow-wave uniform strip configuration. It is to be noted that, the dimensions of the slots etched on the grounds are to be optimized in line with the operating frequencies to achieve commendable matching performances. Simulations are carried out to validate this proposition. The proposed theory to calculate the input characteristics of CPW-fed strip structures in [4] is used to predict the resonance of the modified radiator with the zigzag monopole acting as an excitation to the slot. Later, in order to investigate the robustness of this analytical concept, the width of the zigzag monopole is modified by using a tapered form and the mathematical formulations are modified as well. The modified theory is then validated with the simulations and the measurements of the corresponding structure. The work thus justifies the suitability of the approach which considers the strip radiator as an equivalent uniform rectangular monopole with a modified propagation constant based on the geometrical changes incurred upon it.

Section 2 of this document presents the mathematical formulation, followed by the results and their validations in Sec. 3. The concluding comments along with the acknowledgements are added thereafter in the article.

\section{Mathematical Formulation}

In Fig. 1 the zigzag structure, having uniform width as proposed in [4], is used as an excitation to a wide square slot etched out on the CPW ground plane as shown. The dimensions of the slot are optimized through parametric variations and the corresponding values are provided in Tab. 1. It is noted that, the strip structure acts as a monopole with a height of $H=\lambda_{\mathrm{g}} / 4$ as its electrical length, where 


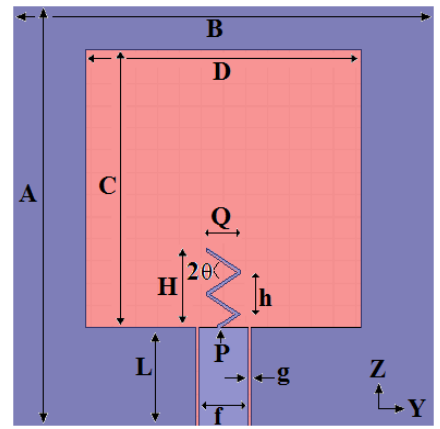

Fig. 1. CPW-fed zigzag monopole exciting a wide-slot (Structure I).

\begin{tabular}{|c|c|}
\hline Parameter Name & Optimal Value (in mm) \\
\hline$A$ & 40 \\
\hline$B$ & 40 \\
\hline$L$ & 10 \\
\hline$f$ & 4.46 \\
\hline$g$ & 0.3 \\
\hline$H$ & 7 \\
\hline$h$ & 4 \\
\hline$Q$ & 3 \\
\hline$C$ & 25 \\
\hline$D$ & 25 \\
\hline
\end{tabular}

Tab. 1. Dimensions for the structure shown in Fig. 1.

$2 \pi / \lambda_{\mathrm{g}}$ is the propagation constant along the strip structure corresponding to the frequency of operation. Accordingly it is observed that, the dimensions of the square slot correspond to an approximate electrical length of $\lambda_{\text {eff }} / 2$, where $\lambda_{\text {eff }}$ is the effective wavelength computed using the effective dielectric constant of the substrate for good matching performances.

The zigzag excitation is modeled as in [4], as an array of two constituent monopoles [3] with modified propagation constants, being fed from the same source. Equations (1-3) are used to calculate the input impedance of the proposed structure (Fig. 1) at P:

$R_{11}=R_{12}=\frac{15 K}{\sin \theta \sqrt{\varepsilon_{\mathrm{eff}}}}$

$\left[\begin{array}{l}\sin \left(2 \beta_{\bmod } H\right)\left\{\operatorname{Si}\left(u_{2}\right)-\operatorname{Si}\left(v_{2}\right)-2 \operatorname{Si}\left(v_{1}\right)+2 \operatorname{Si}\left(u_{1}\right)\right\} \\ -\cos \left(2 \beta_{\bmod } H\right)\left\{2 \mathrm{Ci}\left(v_{1}\right)-2 \operatorname{Ci}\left(u_{0}\right)-\operatorname{Ci}\left(u_{2}\right)+2 \operatorname{Ci}\left(u_{1}\right)-\operatorname{Ci}\left(v_{2}\right)\right\} \\ +4 \mathrm{Ci}\left(u_{0}\right)-2 \mathrm{Ci}\left(v_{1}\right)-2 \mathrm{Ci}\left(u_{1}\right)\end{array}\right]$,

$X_{11}=X_{12}=-\frac{15 K}{\sin \theta \sqrt{\varepsilon_{\text {eff }}}}$

$\left[\begin{array}{l}\sin \left(2 \beta_{\bmod } H\right)\left\{2 \operatorname{Ci}\left(v_{1}\right)-\operatorname{Ci}\left(u_{2}\right)\right. \\ \left.-2 \operatorname{Ci}\left(u_{1}\right)+\operatorname{Ci}\left(v_{2}\right)\right\}-\cos \left(2 \beta_{\bmod } H\right)\left\{2 \operatorname{Si}\left(v_{1}\right)-2 \operatorname{Si}\left(u_{0}\right)\right. \\ \left.-\operatorname{Si}\left(u_{2}\right)-\operatorname{Si}\left(v_{2}\right)+2 \operatorname{Si}\left(u_{1}\right)\right\}+4 \operatorname{Si}\left(u_{0}\right)-2 \operatorname{Si}\left(u_{1}\right)-2 \operatorname{Si}\left(v_{1}\right)\end{array}\right]$.

Here,

$$
\begin{aligned}
& u_{0}=\beta_{\text {mod }}\left(\frac{d}{2}\right), u_{1}=\beta_{\text {mod }}\left(\sqrt{\left(\frac{d}{2}\right)^{2}+H^{2}}-H\right), \\
& v_{1}=\beta_{\text {mod }}\left(\sqrt{\left.\left(\frac{d}{2}\right)^{2}+H^{2}+H\right),}\right. \\
& u_{2}=\beta_{\text {mod }}\left(\sqrt{\left(\frac{d}{2}\right)^{2}+(2 H)^{2}}+2 H\right), \\
& v_{2}=\beta_{\text {mod }}\left(\sqrt{\left(\frac{d}{2}\right)^{2}+(2 H)^{2}}-2 H\right), \\
& Z_{\mathrm{P}}=\frac{Z_{11}+Z_{12}}{2} .
\end{aligned}
$$

$Z_{11}$ and $Z_{12}$ are the self and mutual impedances of the constituent monopoles which model the proposed zigzag structure. Both the impedances may be computed by using the same expressions as in (1) and (2) with proper values of $d$, which is the gap between the equivalent constituent monopoles. The modified propagation constant of the structure is also formulated as [4],

$$
\begin{gathered}
v_{\mathrm{zp}}=c \sin \theta, \\
\beta_{\mathrm{mod}}=\frac{2 \pi}{\lambda_{\mathrm{g}}}
\end{gathered}
$$

where $c$ is the velocity of light computed using the effective dielectric constant of the substrate.

$$
\lambda_{\mathrm{g}}=\frac{v_{\mathrm{zp}}}{f} \text { and } \omega=\frac{f}{2 \pi} .
$$

The input impedance thus calculated at $\mathrm{P}$ can be translated over the length $L$ using (6-7) to calculate the input reflection coefficient of the structure

$$
\begin{gathered}
Z_{\mathrm{IN}}=R_{0}\left[\frac{Z_{\mathrm{P}}+\mathrm{j} R_{0} \tan (\beta L)}{R_{0}+\mathrm{j} Z_{\mathrm{P}} \tan (\beta L)}\right], \\
S_{11}=20 \log _{10}\left|\frac{Z_{I \mathrm{~N}}-50}{Z_{\mathrm{IN}}+50}\right| .
\end{gathered}
$$

When a thin radiator is placed at the center of a slot of width $\lambda_{\text {eff }} / 2$ as shown in Fig. 2 the shunt admittance appearing at the position of the antenna is zero. This is so because it sees two quarter wave short circuited transmission lines at the two sides that offer zero admittance. Therefore the input characteristics of the radiator will not change from its standalone monopole characteristics.

Figure 3 represents the modified structure where the width of the zigzag monopole is tapered to study the characteristics for a shape when the propagation constant is conceived to undergo a gradual change. The values of $C$ and $D$ are changed to $23 \mathrm{~mm}$ to obtain the best matching condition; the width of the zigzag structure $Q$ undergoes a gradual tapering - which increases the value of $Q, 1.5$ times in each turn. The final value of $Q$ remains $3 \mathrm{~mm}$ as before. 


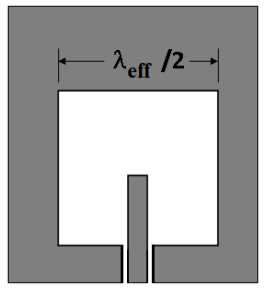

Fig. 2. CPW-fed wide slot structure conceived as terminated by two short-circuited transmission lines.

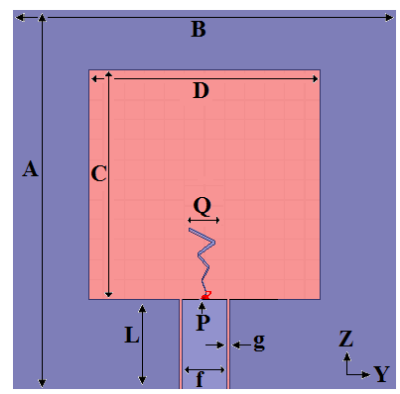

Fig. 3. Proposed CPW-fed tapered zigzag monopole exciting a wide slot (Structure II)

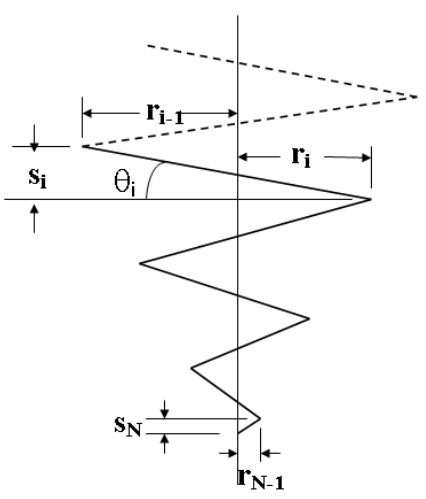

Fig. 4. Mathematical model of the tapered shape.

The gradual change of the propagation constant calls for a modeling of the effective value for the same. For the purpose, the tapered zigzag monopole is modeled mathematically as depicted in Fig. 4, and the calculation of the modified propagation constant follows.

For the $i^{\text {th }}$ arm, let $r_{i}$ and $s_{i}$ be the dimensions as shown in the figure. Assuming $k_{\text {eff }}=2 \pi / \lambda_{\text {eff }}$ to be the propagation constant along the arm, the propagation constant $\beta_{i}$ along the axis is given by

$$
\beta_{i}=\frac{k_{\mathrm{eff}}}{\sin \theta_{i}}=k_{\mathrm{eff}} \frac{\sqrt{\left(r_{i}+r_{i-1}\right)^{2}+s_{i}^{2}}}{s_{i}} .
$$

The expression for average propagation constant $\beta_{\mathrm{av}}$ along the axis is given by

$$
\beta_{\mathrm{av}}=\frac{\sum_{i=1}^{N} s_{i} \beta_{i}}{\sum_{i=1}^{N} s_{i}}=k_{\mathrm{eff}} \frac{\sum_{i=1}^{N-1} \sqrt{\left(r_{i}+r_{i-1}\right)^{2}+s_{i}^{2}}+\sqrt{r_{N=1}^{2}+s_{N}^{2}}}{\sum_{i=1}^{N} s_{i}} .
$$

The modified propagation constant obtained from (9) can be used in (1-3), (6), (7) to predict the input characteristics of the proposed radiator in Fig. 3.

\section{Results and Analysis}

Structures I and II are simulated and fabricated on Arlon AD430 substrate material - with a dielectric constant of 4.3 and having a thickness of $0.762 \mathrm{~mm}$. Simulations are carried out on ANSYS HFSS [7] and the measured results are compared with the same as well as with the theoretical predictions. The feed width $f$ was chosen to be $4.46 \mathrm{~mm}$ to represent a $50 \Omega$ transmission line with the help of IE3D line gauge calculator [8]. Figure $5(a-b)$ represents the fabricated prototypes followed by the $\mathrm{Z}$ and $\mathrm{S}$-parameter results in Fig. 6 and 7, respectively. In Fig. 6(a-b) the theoretical input impedances of the structures are compared to their measured counterparts and both the resistive and the reactive components are seen to be in good agreement with each other. At higher frequencies, some deviations are observed between the theory and the measurements - however as the induced EMF method is suitable for dipole radiators whose electrical lengths lie between the ranges of $0.2 \lambda$ to $0.7 \lambda$ ( $\lambda$ being the wavelength corresponding to the frequency of operation) [9] - this discrepancy is expected and yet, at and around resonance the agreement between the results are commendable.

For Structure I the predicted resonance was at $4.49 \mathrm{GHz}(4.54 \mathrm{GHz}$ as per the measurements, error in prediction being $1.1 \%$ ) and for Structure II the predicted resonance was at $4.76 \mathrm{GHz}(4.67 \mathrm{GHz}$ as per the measurements, error in prediction being $1.8 \%$ ), respectively.

From Fig. 7 it is evident that the theoretical analysis can accurately predict the resonance frequencies of the proposed structures. In terms of bandwidth, the theoretical results present better match with those of the measurements as compared to the simulations. This validates the suitability of the proposed expressions and justifies the use of

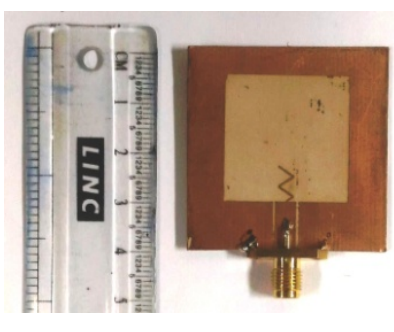

(a)

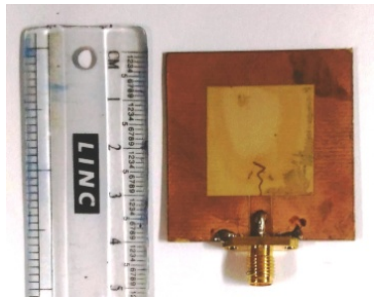

(b)

Fig. 5. Fabricated prototypes of (a) Structure I and (b) Structure II, respectively. 
propagation constant modification based modeling of strip monopole radiators.

Figure 8(a-b) represents the simulated surface current distributions of the uniform and tapered zigzag structure at their frequencies of resonances respectively. The observation that the distribution has minimal surface current at the

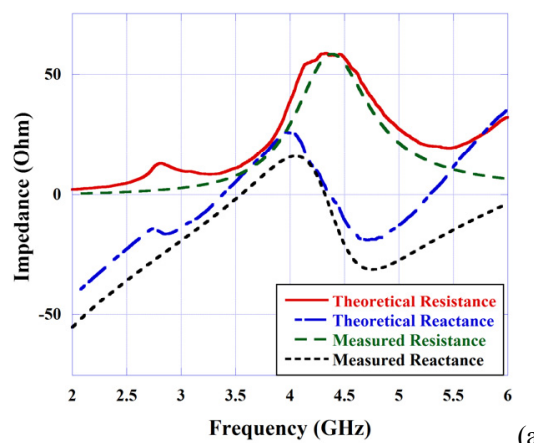

(a)

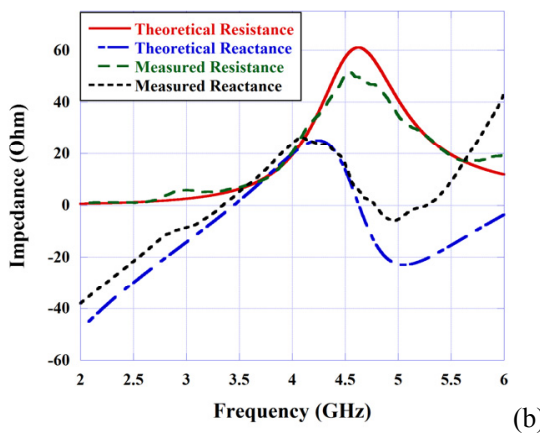

Fig. 6. Comparison between the theoretical and measured results of $Z_{11}$ for (a) Structure I and (b) Structure II, respectively.

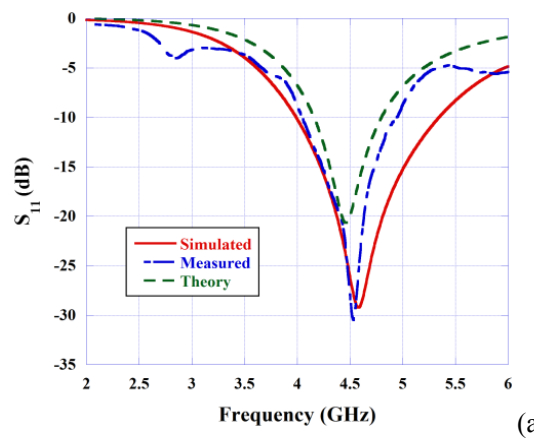

(a)

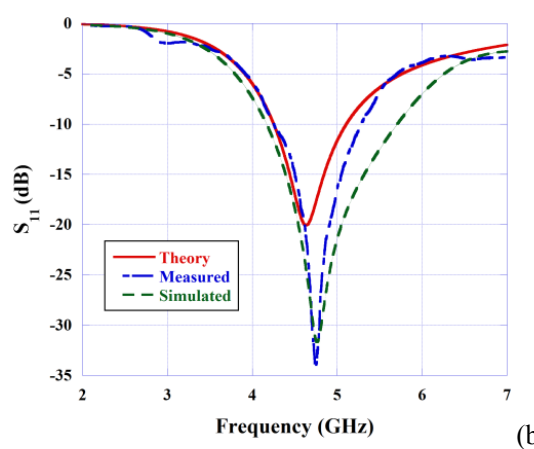

(b)

Fig. 7. Comparison between the simulated, theoretical and measured results of $\mathrm{S}_{11}$ for (a) Structure $\mathrm{I}$ and (b) Structure II, respectively.

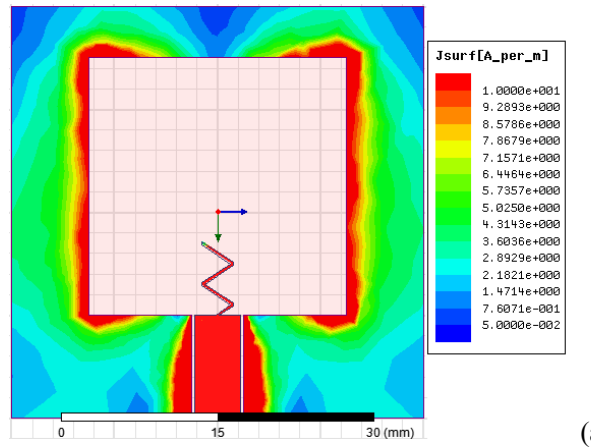

(a)

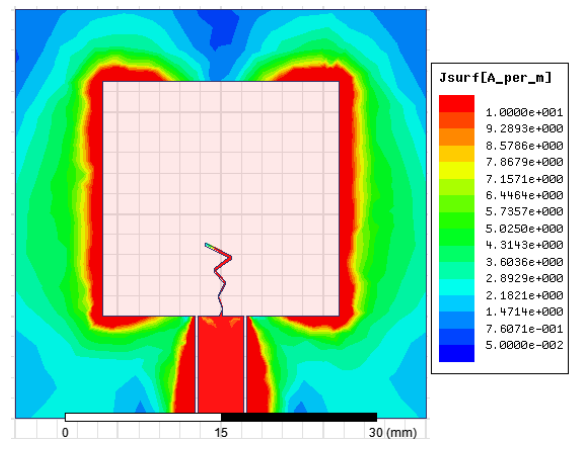

(b)

Fig. 8. Simulated surface current distributions for (a) Structure I and (b) Structure II, respectively.

lower edge regions of the rectangular slot where the feed is connected to the exciting monopole justifies the authors' assumption to consider the shunt admittance appearing at the feeding position $\mathrm{P}$ of the monopole being zero as elaborated in the previous section (Fig. 2).

Figure $9(\mathrm{a}-\mathrm{d})$ represents a comparison between the simulated and measured radiation patterns of both the structures at their frequencies of resonance. The co-polar patterns are almost identical for both simulation and measurements. Some discrepancies are observed in the magnitudes of cross-polar radiation which may be accounted for the possible fabrication inaccuracies. It may also be noted that, even with the differences observed between the simulated and measured cross-pol patterns - in all cases, the difference between the co and cross radiations are sufficiently large to suggest the utility of the proposed structures for practical operations. The frequencies of resonance for structure I and II were found to be $4.54 \mathrm{GHz}$ and 4.67 GHz, respectively. In [4] the stand alone CPW-fed zigzag structure could provide a gain of $0.672 \mathrm{~dB}$. However, as expected - by using the slot as the aperture being excited by the strip, sufficient gain enhancement could be achieved. Measured gains for Structure I and II at $4.54 \mathrm{GHz}$ and $4.67 \mathrm{GHz}$ were found to be $2.93 \mathrm{~dB}$ and $3.19 \mathrm{~dB}$ respectively. The measured and simulated gain values are included in Fig. 10.

It may also be noted that the tapering of the zigzag structure was mostly motivated to analyze the characteristics of a structure supporting a gradual propagation constant modification. However, such an operation led to an enhanced matching performance of the input reflection coefficient and an increased value of antenna gain, as observed from the measurements. 


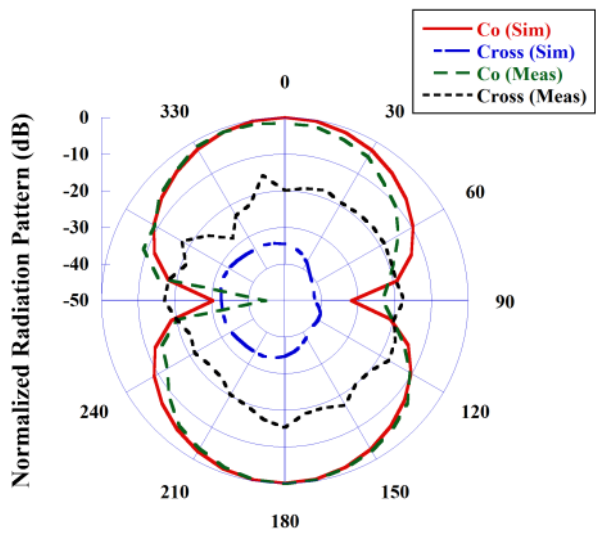

(a)

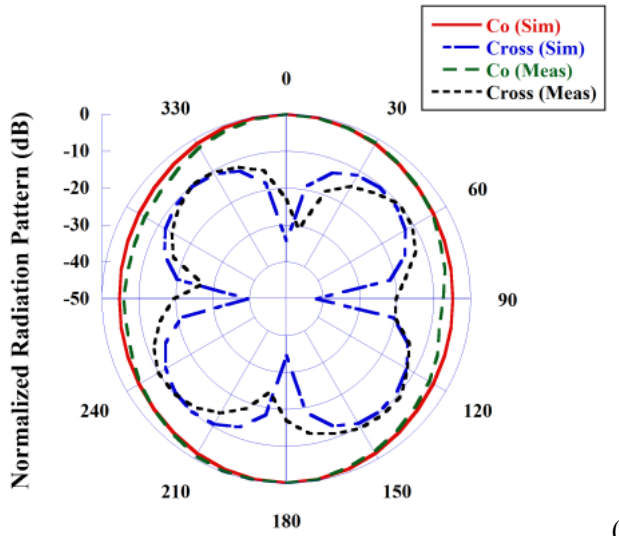

(b)

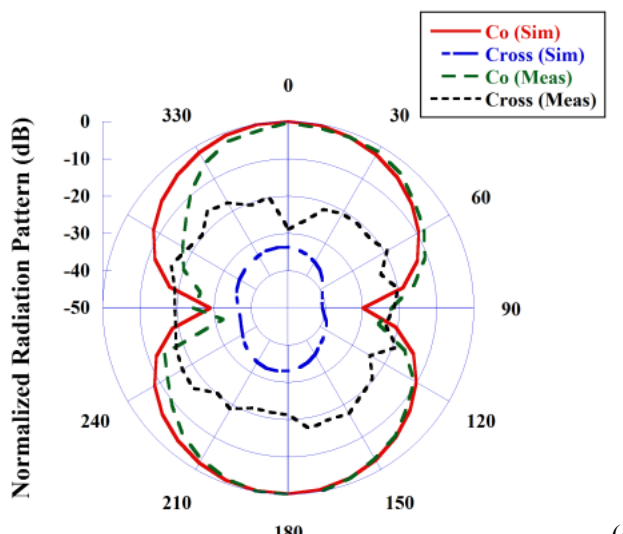

(c)

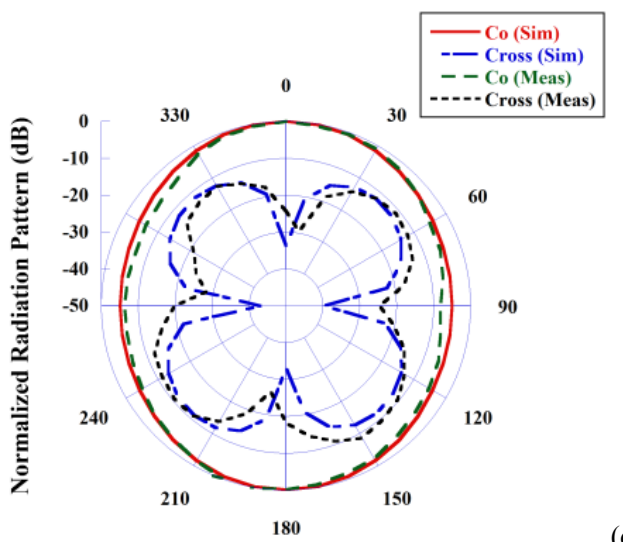

Fig. 9. Co and cross-polar radiation patterns for (a) XZ-plane, Structure I at $4.54 \mathrm{GHz}$, (b) XY-plane, Structure I at $4.54 \mathrm{GHz}$, (c) XZ-plane, Structure II at $4.67 \mathrm{GHz}$ and (d) XY-plane, Structure II at $4.67 \mathrm{GHz}$, respectively.

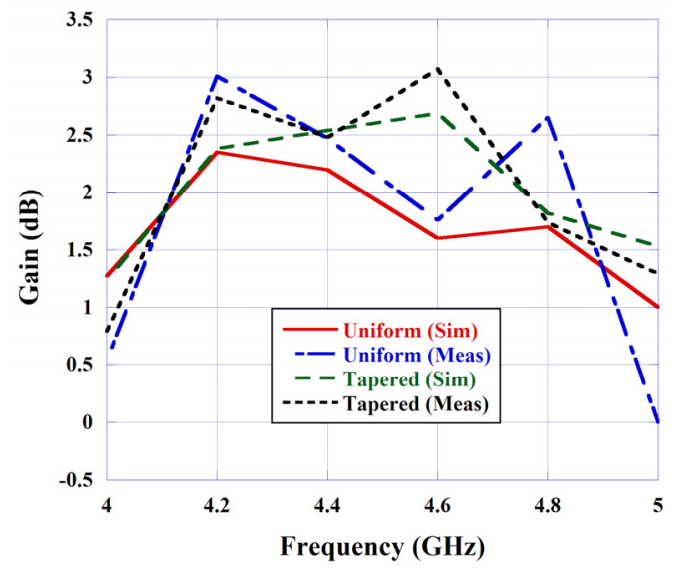

Fig. 10. Simulated and measured gain plots for the uniform and tapered structures.

\section{Conclusions}

The work presents a theoretical analysis of the CPWfed zigzag monopole radiator, which excites a wide square slot on the CPW-grounds for gain enhancement purposes. The theoretical analysis conceived the zigzag feed as an equivalent array of two strip monopoles with modified propagation constants, fed from the same source and the input reflection coefficient results are predicted and validated by simulations and measurements. Later, the zigzag feed was tapered to conceive a structure supporting gradual propagation constant modification and appropriate expressions are proposed for the calculation of the effective value. Again the proposed structure is analyzed as an array of two constituent monopoles with modified propagation constants, which are fed from the same source and the input characteristics are predicted. The results are validated by simulations and measurements along with the radiation characteristics. Measured gains of the structures at their frequencies of resonance are reported to justify the ability of their practical operations.

\section{Acknowledgments}

This work is supported by CSIR, Govt. of India (ack. no.: 143126/2K17/1 and File No. 09/096(0925)/2018EMR-I, dated: 02/05/2018).

\section{References}

[1] LAOHAPENSAENG, C., FREE, C., ROBERTSON, I. D. Simplified analysis of printed strip monopole antenna fed by a CPW. In Proceedings of the Asia-Pacific Microwave Conference. Suzhou (China), 2005, p. 1-4. DOI: 10.1109/APMC.2005.1606956

[2] BANERJEE, A., BANDYOPADHYAY, A. K. Theoretical investigation on the input impedance of a CPW-fed strip monopole antenna. Microwave and Optical Technology Letters, 2017, vol. 59, no. 2, p. 346-348. DOI: 10.1002/mop.30287 
[3] BANERJEE, A., CHATTERJEE, S., GUPTA, B., et al. Theoretical investigation on input characteristics of CPW-fed wide rectangular monopole structures. In IEEE International Conference on Antenna Innovations and Modern Technologies for Ground, Aircraft and Satellite Applications (iAIM). Bangalore (India), 2017. DOI: 10.1109/IAIM.2017.8402614

[4] BANERJEE A., PATRA K., CHATTERJEE S., et al. Theoretical investigations on the resonance characteristics of $\mathrm{CPW}$-fed miniaturized strip monopole antennas. Radioengineering, 2018, vol. 27, no. 4, p. 948-955. DOI: 10.13164/re.2018.0948

[5] SIM, C. Y. D., CHEN, H. D., CHIU, K. C., et al. Coplanar waveguide-fed slot antenna for wireless local area network/worldwide interoperability for microwave access applications. IET Microwaves, Antennas and Propagation, 2012, vol. 6, no. 14, p. 1529-1535. DOI: 10.1049/iet-map.2012.0174

[6] HU, L., HUA, W. Wide dual-band CPW-fed slot antenna. Electronics Letters, 2011, vol. 47, no. 14, p. 789-790. DOI: 10.1049/el.2011.0909

[7] Ansoft Corp HFSS v.13

[8] Zealand Corp IE3D v.10

[9] ESTARKI, M. D., VAUGHAN, R. G. Theoretical methods for the impedance and bandwidth of the thin dipole. IEEE Antennas and Propagation Magazine, 2013, vol. 55, no. 01, p. 62-81. DOI: 10.1109/MAP.2013.6474485

\section{About the Authors ...}

Amartya BANERJEE (corresponding author) was born in September, 1992. He received his M.Tech. from IIEST, Shibpur in 2016. His research interests include microwave and antenna design techniques, circuit theoretical analysis and signal processing.

Sayan CHATTERJEE was born in Kolkata, India, in 1980. Presently, he is an Associate Professor at the Department of Electronics and Telecommunication Engineering, Jadavpur University. His research interest includes microwave and millimeter wave antennas, passive devices and slotted array antenna

Bhaskar GUPTA was born in Kolkata, India, in 1960. He received the B.E.Tel.E., M.E.Tel.E., and Ph.D. degrees from Jadavpur University, Kolkata, India, in 1982, 1984, and 1996, respectively. He is currently a Professor in the Department of Electronics and Telecommunications Department, Jadavpur University, where he has been teaching since 1985 . He has published about 350 research articles in refereed journals and conferences and coauthored three books on advanced research topics. His present area of interest is planar antennas, dielectric resonator antennas, wearable antennas, photonic band gap materials, computational electromagnetic, MEMS design and application of soft computing techniques in microwave engineering and antennas. He has been named in the 2009 edition of Marquis' Who's Who of the World.

Anup Kumar BANDYOPAHDYAY is a retired Professor from the Dept. of ETCE, Jadavpur University, Kolkata. His research interests include microwave and antenna engineering, circuit theory and program proving. 